\title{
Clinical and Immunologic Effects of Allergen-Specific Sublingual Immunotherapy in a Canine Model of Atopic Dermatitis: A Double Blind, Randomized, Controlled Study
}

\section{Marsella $\mathbf{R}^{*}$ and Ahrens $\mathrm{K}$}

Department of Small Animal Clinical Sciences, College of Veterinary Medicine, University of Florida, USA

\begin{abstract}
Sublingual Allergen Specific Immunotherapy (SLIT) has been advocated for the treatment for allergies. Controlled studies are difficult to perform in humans due to the variety of allergies, diet, and allergenic exposure. This prospective, randomized, controlled study evaluated clinical and immunological effects of one year of SLIT using an experimental model of atopic dermatitis in dogs. Eighteen Beagles, sensitized to dust mites, timothy grass and ragweed were divided into control $(n=6$, vehicle) and active $(n=12,3$ allergens) groups. Allergen challenge and scoring of clinical signs was done before and at the end of one year of SLIT. Blood was drawn at baseline, 4,8, and 12 months of SLIT and 2 months after stopping SLIT to measure allergen-specific IgE, IL-10, and TGF-beta.

After 12 months of SLIT, ANOVA showed significant decrease of clinical scores for both groups $(p<.0001)$ but no significant differences between groups. T tests within each group comparing pre vs. post treatment scores showed statistically significant decrease in the control $(p=0.042)$ and in the SLIT group $(p=0.00027)$. Effect size using Cohen's $\mathrm{d}$ was 1.182 for control and 2.1 for allergen group. Thus, decrease from baseline to post-treatment was nearly twice as large in the allergen as in the control group.

Mixed results were found for allergen-specific $\lg E$ with significant decrease for dust mites $(p=0.0242)$ and increase for ragweed $(p=0.0074)$ at the end of the study. SLIT induced significant increase of TGF-beta $(p=0.03)$ and IL-10 $(p=0.0009)$ after ragweed stimulation compared to baseline and to the control group. TGF-beta increase abated after SLIT discontinuation results consistent with SLIT induced T regulatory response. Interestingly a significant increase for IL-10 after timothy stimulation was seen for both groups at the end of study $(p<.0001)$.It is concluded that this experimental model is useful to investigate treatments for atopic dermatitis and their immunologic effects.
\end{abstract}

Keywords: Atopic dermatitis; Dogs; Sublingual; Allergen-specific immunotherapy

Abbreviations: SLIT: Sublingual Allergen Specific Immunotherapy; ASIT: Allergen specific immunotherapy; AD: Atopic Dermatitis; SCIT: Sub Cutaneous Immunotherapy; FDA: Federal Drug Administration

\section{Introduction}

Allergen specific immunotherapy (ASIT) is currently considered the best long term approach to manage environmental allergies in both human and veterinary medicine [1-3] when allergen avoidance is not feasible. Currently, ASIT is indicated for the treatment of allergic rhinitis, allergic asthma and hymenoptera-induced anaphylaxis. In human medicine sublingual immunotherapy (SLIT) has been used with good success for allergic conjunctivitis, rhinitis and asthma [4-6]. An advantage of SLIT over the traditional route of subcutaneous injections is the easy administration and the excellent tolerability [7]. SLIT will also be more cost-effective over time with increased usage than the traditional subcutaneous injections as it does not require frequent office visits. More recently, there have been reports of beneficial effects of ASIT for atopic dermatitis (AD) although this approach is not routinely used for patients with only cutaneous disease [8-11].

Administration of the allergens during ASIT via the subcutaneous (subcutaneous immunotherapy-SCIT) and the sublingual (SLIT) route has been well established to induce tolerance. Modulation of T regulatory cells is believed to be important in clinical response [12]. Some allergens, such as grass pollen, house dust mite can be delivered through either route, whereas others like venoms are only delivered subcutaneously [13]. SLIT has been commercially available and routinely used in Europe with satisfactory safety profile [14]. There is no Federal Drug Administration (FDA) approved formulation for SLIT in
United States. Despite the lack of an FDA-approved SLIT formulation, SLIT prescribers have significantly increased from $5.9 \%$ in 2007 to $11.3 \%$ in 2011 [15] and SLIT is also being studied for food allergies [16]. On the basis of available literature, SLIT is certainly indicated in allergic rhinitis and asthma which is associated with rhinitis in both adults and children [17]. SLIT is currently accepted as an alternative to injections because of its satisfactory safety profile. Its efficacy and safety in rhinitis and asthma in children has been confirmed with clinical trials [6]. Atopic dermatitis is also being considered as a promising field for use of SLIT [15]. A meta-analysis, recently published, reported moderate-level evidence for the efficacy of SLIT against AD but the authors noted that the findings were based on an analysis of a small number of randomized controlled trials, with considerable heterogeneity among trials [18]. Controlled studies in human medicine that are able to minimize the variability of allergies, environmental exposure and concurrent drug treatments are difficult to complete. For these reasons access to an experimental model for $\mathrm{AD}$ could prove to be beneficial.

*Corresponding author: Rosanna Marsella, Department of Small Animal Clinical Sciences, College of Veterinary Medicine, University of Florida, Gainesville, Florida USA, Tel: +1-352-2780742; Fax: +1-352-392 6125; E-mail: Marsella@ufl.edu

Received September 16, 2013; Accepted December 16, 2013; Published December 20, 2013

Citation: Marsella R, Ahrens K (2013) Clinical and Immunologic Effects of Allergen-Specific Sublingual Immunotherapy in a Canine Model of Atopic Dermatitis: A Double Blind, Randomized, Controlled Study. J Allergy Ther 4: 157. doi:10.4172/2155-6121.1000157

Copyright: (c) 2013 Marsella R, et al. This is an open-access article distributed under the terms of the Creative Commons Attribution License, which permits unrestricted use, distribution, and reproduction in any medium, provided the original author and source are credited. 
Dogs naturally develop $\mathrm{AD}$, with characteristics that are clinically and immunologically similar to the human counterpart $[19,20]$. Dogs are closer to humans than mice and share the same environment with humans thus making them an ideal choice for a model. An experimental model for $\mathrm{AD}$ has been validated using atopic Beagles [21-23]. Symptoms of AD in both species include recurrent pruritic dermatitis affecting the flexural surfaces, periocular, perioral, axillary, and inguinal areas. It is common for patients to have sensitization to multiple allergens. Amongst them, house dust mites, timothy grass and ragweed are commonly responsible for flare ups of $\mathrm{AD}$ in both dogs and humans. Thus, the purpose of the present study was to evaluate the efficacy of SLIT in a controlled and standardized manner using an experimental canine model for AD. Our study was designed as prospective, vehicle-controlled trial in which dogs were specifically sensitized to 3 allergens of interest (ragweed [RW], timothy grass [TG] and dust mites $[\mathrm{DM}])$ and were kept in an environment where allergen exposure could be strictly controlled and dosed. We aimed to evaluate clinical improvement scoring the clinical signs using a validated clinical scoring system called CADESI (Canine Atopic Dermatitis Extent and Severity Index) [24]. The hypotheses tested in our study were that SLIT would decrease severity of clinical signs as measured by CADESI and that the clinical improvement would be associated with a decrease in allergen specific IgE and an increase in T regulatory cytokines such as Interleukin-10 (IL-10) and Transforming Growth Factor-beta (TGF- $\beta$ ) by peripheral blood mononuclear cells (PBMC).

\section{Materials and Methods}

All procedures of this study had been approved by the Institutional Animal Care and Use Committee of the University of Florida.

\section{Experimental design}

The study was designed as a prospective, vehicle controlled study that lasted a total of 18 months. The first 4 months were used for sensitization, 12 months were used for SLIT and additional monitoring was done for 2 months past the completion of SLIT. During the study, both immunological and clinical monitoring was done. See Figure 1 for time table of events.

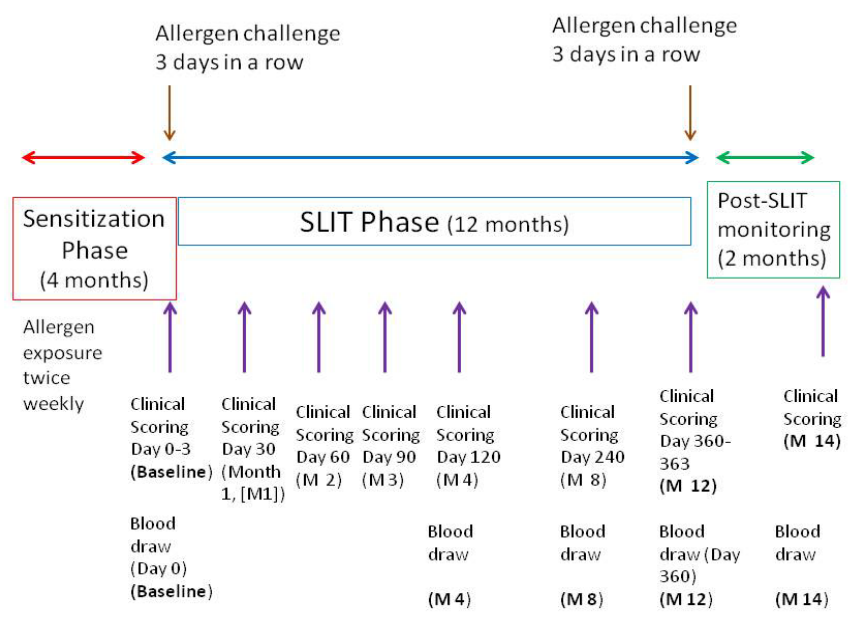

Figure 1: This study included various phases: 4 months of sensitization, 12 months of sublingual immunotherapy (SLIT), and 2 months of monitoring post SLIT.

\section{Animals}

Eighteen atopic Beagles were used. These dogs have been previously sensitized epicutaneously to Dermatophagoides farinae and were known to develop pruritic dermatitis clinically and histologically compatible with $\mathrm{AD}$ upon allergen exposure to DM. No respiratory or ocular signs were observed in this model upon allergen challenge. For this study the dogs were additionally sensitized to two other allergens (Timothy Grass [TG], and Ragweed [RW]). We selected these two additional allergens besides dust mites so that we could provide a model that would incorporate the most common allergies $[25,26]$ and test the efficacy of a SLIT preparation that was a mixture of frequently used allergens.

\section{Housing conditions}

All dogs were housed in a research facility at the University of Florida. All dogs were on the same diet throughout the study and housed in the same temperature and humidity controlled research facility (air conditioning was set at $18 \mathrm{C}$ and the average humidity recorded was 68\%). Dogs were housed in individual cement runs that were cleaned daily using high temperature and high pressure wash. Stuffed toys, carpets, soft bedding or anything that could trap dust were not allowed in these runs. Walls and air filters were checked to ensure the absence of mites in the environment in between challenges. Results were always below the detection level of the test used (MITE-T-Fast ${ }^{\text {tw }}$ Allergen Detection System, Aveho Biosciences, CA). All personnel interacting with the dogs used protective clothing to avoid introduction of allergen from outdoor environment.

\section{Sensitization phase}

Sensitization procedure: All atopic Beagles had been sensitized to HDM as previously described [27]. Briefly, sensitization was done with Dermatophagoides farinae pure culture (RMB83M, $>99 \%$ pure whole bodied natural milled D. farinae, Greer Laboratories, Lenoir, NC) when puppies were 2 months old. One gram $\mathrm{D}$. farinae culture was mixed with sterile saline $(2.5 \mathrm{ml})$ to create a thick paste $(400 \mathrm{mg} / \mathrm{ml})$ and each puppy received $0.3 \mathrm{ml}$ of $\mathrm{D}$. farinae $(133 \mathrm{mg} /$ dose $)$ twice weekly for a total of 12 weeks. The thick paste was applied to small areas of untreated skin in the axillae or inguinal areas using a soft brush.

For the present study all atopic Beagles were also sensitized to ragweed and timothy grass. Sensitization involved biweekly epicutaneous application of timothy and short ragweed allergens for a total of 4 months. Allergenic extract at $100 \mathrm{mg} / \mathrm{ml}$, diluted to $50 \%$ in glycerine (Nelco Laboratories Inc, Deep Park, NY) was applied with dropper vials, $2.6 \mathrm{mls}(133 \mathrm{mg})$ to axillary area, alternating right and left side. Verification of successful sensitization was done by detection of allergen specific IgE on serology and development of clinical signs upon allergen challenge. Procedure for allergen challenge is described in appropriate section.

Immunological monitoring of atopic Beagles during sensitization phase: Blood samples $(4 \mathrm{ml})$ were taken from the atopic beagles at baseline and every 2 months during the sensitization phase to measure allergen-specific IgE using Allercept (Heska, Fort Collins, $\mathrm{CO})$. According to this test results are expressed in ELISA Allergy units (EA).In this test values above 150 are considered positive and compatible with allergic sensitization.

Clinical monitoring: Clinical evaluation was done using a modified version of the validated Canine Atopic Dermatitis Extent and Severity Index Score 03 (CADESI) [23]. The two modifications in relation to CADESI-03 were that papules were added as a clinical sign 
and that the scores for each sign ranged from 0 to 3 rather than from 0 to 5 [28]. The dog's body was divided into sections, each of which received a score based on the clinical signs evaluated. The total score was the sum of all body sites. Clinical signs evaluated included diffuse erythema, erythematous macules, papules, excoriations and alopecia. The total score is calculated by adding the score of various body regions and clinical signs. The total maximum possible score was 150 . Total scores were used in the statistical analyses.

Clinical scoring of the atopic Beagles was done at baseline, 1, 2, 3, 4,8 , and 12 months of SLIT. At the beginning and at the end of both sensitization and immunotherapy phase dogs were epicutaneously challenged with allergens. Clinical signs were scored daily during the weeks of allergen challenges. Each allergen challenge lasted5 days (see below for more details on allergen challenges).

Allergen challenge procedure at the end of sensitization phase: Atopic Beagles were epicutaneously challenged with $50 \mathrm{mg}$ of timothy and ragweed mix. The reason not to challenge them with HDM too was to avoid masking clinical signs with the already known allergy that they had for HDM. The allergens were applied epicutaneously with a brush on glabrous areas such as the inguinal area three days in a row and monitored for a total of 5 days to detect development of dermatitis.

\section{SLIT phase}

SLIT protocol and group allocation: Once all atopic Beagles were sensitized to all allergens they were randomly assigned to either vehicle group (control) or active ingredient (SLIT) group. The investigator evaluating the dogs was blinded to the allocation of the dogs to the two groups. The randomization was done such that $1 / 3$ of the dogs received vehicle or $50 \%$ glycerine $(n=6)$ and $2 / 3$ of the dogs $(n=12)$ received the active ingredients consisting of allergen mixture of HDM $5,000 \mathrm{aq}$, timothy $1-20 \mathrm{w} / \mathrm{v}$ and ragweed $1-20 \mathrm{w} / \mathrm{v}$, in $50 \%$ glycerine, Nelco laboratories). The following schedule was used: 3 squirts $(0.3$ $\mathrm{ml}$ ) sublingually daily in the first month, 6 squirts daily for the second month and 8 squirts daily from the third month until the end of study (total of 12 months).

\section{Immunologic monitoring during SLIT phase}

a. Allergen specific IgE: Blood samples $(12 \mathrm{ml})$ were drawn by jugular venipuncture before beginning SLIT, 4,8 , and 12 months of SLIT and 2 months after stopping SLIT. $4 \mathrm{ml}$ was transferred to EDTA or serum vacutainer tubes, serum collected was stored at $-80^{\circ} \mathrm{C}$ until analyzed by ELISA with an Allercept ${ }^{\circ} 48$-Allergen SE Regional Panel performed by HESKA Veterinary Diagnostic Laboratories (Loveland, CO) to measure allergen-specific IgE. The results were expressed in EA units and included D. farinae, short ragweed and timothy grass.

b. PBMC separation and culture: $8 \mathrm{ml}$ was used for PBMC isolation, stimulation and measurement of IL-10 and TGF- $\beta 1$ using Elisa Assays (R\&D Systems, Minneapolis, MN). Canine blood in EDTA was processed within 1-2 hours for cell culture as described by Strasser et al. [29] 1998 with modifications. Briefly, $6 \mathrm{ml}$ of undiluted room temperature blood was layered over $12 \mathrm{ml}$ of ice cold Lymphoprep ${ }^{\mathrm{mw}}$ (Axis-Shield PoC, Oslo, Norway) in a $50 \mathrm{ml}$ polypropylene tube and centrifuged for 30 minutes at $340 \times \mathrm{g}$. The top layer above the buffy coat was removed and the buffy coat layer transferred to a $15 \mathrm{ml}$ tube where it was washed with Dulbecco's Phosphate Buffer Saline $(\mathrm{D}$-PBS) for 10 minutes at $340 \times \mathrm{g}$. The supernatant was removed and the pellet washed three more times at $300 \times \mathrm{g}$ for
10 minutes with D-PBS. After last wash $2 \mathrm{ml}$ cold ammonium chloride erythrocyte lysis buffer was added and incubated for 10 minutes, then centrifuged 5 minutes at $200 \times$ g. The cells were washed two more times in PBS at room temperature to eliminate residual platelets, and then resuspended in RPMI/10\%FBS. Cell counts were taken using a hemacytometer and assessed for viability with trypan blue exclusion staining.

c. Cell culture set-up: Peripheral blood mononuclear cells were resuspended to $1 \times 10^{7}$ cells per $\mathrm{ml}$, and then $100 \mu \mathrm{l}$ was aliquoted into five separate 96 well plates. Cells rested overnight in a $37^{\circ} \mathrm{C}$, $5 \% \mathrm{CO} 2$ incubator, then $100 \mu \mathrm{l}$ of the following were added diluted in RPMI; RPMI only, $20 \mu \mathrm{g} / \mathrm{ml}(1-100 \mathrm{w} / \mathrm{v})$ of D. farinae $(10,000 \mathrm{AU} / \mathrm{ml})$ in $50 \%$ glycerol, $2 \mathrm{mg} / \mathrm{ml}(1-10 \mathrm{w} / \mathrm{v})$ of short ragweed $(81,000 \mathrm{PNU} / \mathrm{ml}$ or $100 \mathrm{mg} / \mathrm{ml}), 2 \mathrm{mg} / \mathrm{ml}(1-10 \mathrm{w} / \mathrm{v})$ of timothy grass $(74,000 \mathrm{PNU} / \mathrm{ml})$ allergenic extracts (Nelco Labs, Deer Park, NY), or $2 \mu \mathrm{g} / \mathrm{ml}$ of E.coli lipopolysaccharide (Astarte Biologics, Redmond, WA). Cells were incubated for 24 hours, collected, centrifuged for 5 minutes and culture media only transferred to eppendorf tubes that were frozen at $-80^{\circ} \mathrm{C}$ until used in ELISA assays for IL-10 and TGF- $\beta 1$.

d. Elisa Assays for canine IL-10 and TGF- $\beta 1$ : ELISAs were performed according to manufacturer's protocol using Quantikine Canine IL-10 and TGF- $\beta 1$ kits (R \& D Systems, Minneapolis, MN). Culture media for TGF- $\beta 1$ was diluted 1:2 in RPMI media only and activated to immunoreactive TGF- $\beta 1$, then assayed after neutralization. Culture media was undiluted for testing with IL-10. Samples were assayed in duplicate and included normalization controls for each subject's lymphocytes incubated with no allergen and culture media. This result was then subtracted from allergen and control values. Results are presented in $\mathrm{pg} / \mathrm{ml}$.

Allergen challenge at the end of SLIT: $50 \mathrm{mg}$ of crude allergens (mix of HDM, ragweed, timothy grass) were applied epicutaneously for 3 consecutive days. Clinical signs were scored daily for a total of 5 days using the CADESI scoring system. Clinical scoring was done right before allergen challenge ( $0 \mathrm{hr}), 6 \mathrm{hrs}$ after each allergen exposure on the days that allergen was applied (first 3 days), and then every 24 hours for 2 additional days. Following this schedule the evaluations were done at hours $0,6,24,30,48,54,72,96$.

\section{Statistical analysis}

Statistical analysis was performed by using the JMP statistical software (SAS institute). 2-Group x 7-Time REML Analysis of Variance (ANOVA) was used to evaluate differences between groups and over time for total CADESI, allergen specific IgE, IL-10 and TGF- $\beta$. Paired t-test was used to compare the sum of CADESI scores pre vs. post treatment within each group and Cohen's $d$ was used to calculate effect size of treatment for each group. Statistical significance was defined as $\mathrm{P}<0.05$.

\section{Results}

\section{Sensitization phase}

Clinical scores: All atopic beagles developed sensitization to RW and TG as confirmed by increased clinical signs after epicutaneous application of the allergens (Figure 2). An erythematous macular, papular pruritic dermatitis was evident on all dogs within the first 24 hours of allergen exposure. The degree of erythema and pruritus progressively increased over the course of the allergen challenge as 


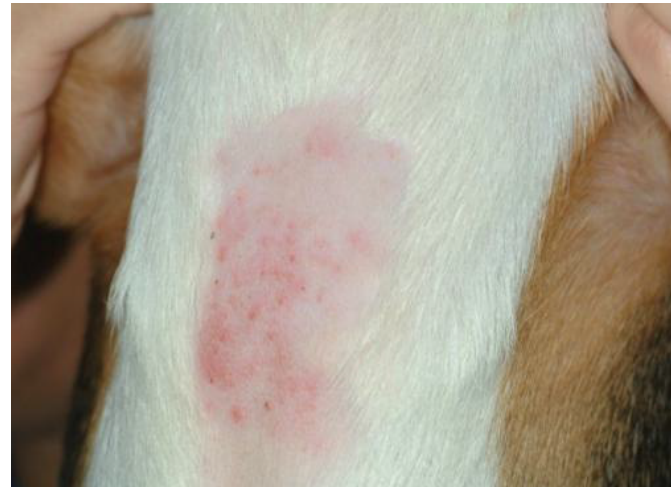

Figure 2: Erythematous macules and papules on the chest of an atopic beagle 6 hours after application of a mixture of timothy grass and ragweed allergen. The chest area of this image would be scored as a 2 (moderate) for erythema, 2 for macules and 3 (severe) for papules. Thus the total score of this specific area would be a 7 . All body areas would be scored with the same system and the total of all body areas would be the total clinical score of the dog.

CADESI at the end of sensitization phase

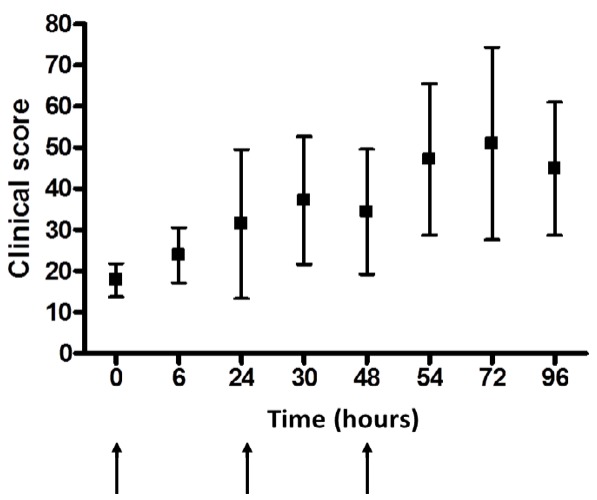

Figure 3: Total clinical scores expressed as CADESI (Canine Atopic Dermatitis Extent and Severity Index) scores at the end of sensitization phase with timothy and ragweed during an allergen challenge. The arrows indicate the times when allergen was applied epicutaneously. Time is expressed in hours. The black square indicates the mean and the bars the standard deviation of the total clinical scores. A progressive increase in clinical scores was observed over the 5 day observation period in response to allergen exposure.

illustrated by the increase of clinical scores (Figure 3). Although some variability was noted in the severity of dermatitis developed by the various dogs, all dogs flared up with dermatitis upon allergen exposure and were therefore considered clinically reactive to the allergens.

Allergen specific IgE: At the end of the sensitization period all dogs developed also allergen specific IgE above the 150 ELISA Allergy Units cut off. Variability among dogs in the intensity of sensitization was noted but all the results were well above the threshold to be considered positive (Figure 4). Allergen specific IgE were higher for dust mites than other allergens. Many dogs (10/18) had allergen-specific IgE over 2,000 ELISA Allergy units for dust mites, which were considered a very high result for atopic dogs.

\section{SLIT phase}

Clinical scores: The results of the first allergen challenge performed with timothy, ragweed mix and dust mites after the successful sensitization and before starting the SLIT phase showed no significant differences between the dogs allocated to the allergen (SLIT group) and the ones allocated to the control group (Figure 5A) reassuring that the group allocation was balanced for severity. ANOVA showed a significant effect of time $(\mathrm{p}<0.0001)$ with both groups increasing their clinical scores during the course of allergen challenge (Figure $5 \mathrm{~A}$ ). At the end of 12 months, in the last allergen challenge similar results were found meaning that both groups increased in severity of scores during the course of challenge (significant effect of time, $p<0.0001$ ) but no significant differences were found between the two groups (Figure $5 \mathrm{~B})$. Due to small number of dogs used in this study and the variability of severity of disease among individual dogs we then evaluated how scores changed within each group so that each dog would be compared to his/her own baseline. At the end of 12 month period the average decrease of the sum of clinical scores was $33 \%$ in the control and $48 \%$ in the SLIT group. When CADESI scores during allergen challenge were added and compared pre vs post treatment within each group a statistically significant decrease of total scores was found both in the control group ( $\mathrm{p}=0.042$, Figure $5 \mathrm{C}$ ) and in the SLIT group ( $\mathrm{p}=0.00027$, Figure 5D). Effect size using Cohen's $d$ was 1.182 for control and 2.1 for SLIT group meaning that the decrease was nearly twice as large in the SLIT compared to the control group.

Individual dogs assigned to the SLIT group had remarkable clinical improvement despite allergen exposure as shown by dog in Figure 6 . None of the control dogs had the same clinically striking response.

When the total scores of the two groups were compared over the course of the 12 months of SLIT without any allergen challenge, ANOVA showed no statistically significant effect of group or group $x$ time interaction meaning that the severity of dermatitis was not significantly different between groups when not challenged with allergens. A significant effect of time was detected between baseline and month 12 ( $\mathrm{p}<.0001)$ (Figure 7) meaning that the clinical scores for both groups significantly decreased at month 12 . It is important to note that the control group was not exposed to any allergens for the 12 months of the SLIT phase, which possibly lead to a progressive improvement of the dermatitis just because of lack of allergen exposure.

Adverse effects: No systemic adverse effects were noted in this study. Two dogs in the study had to follow a slower increasing protocol (delay of a couple of weeks in the induction phase) to minimize pruritus and erythema. All dogs were able to reach the maintenance dose and no

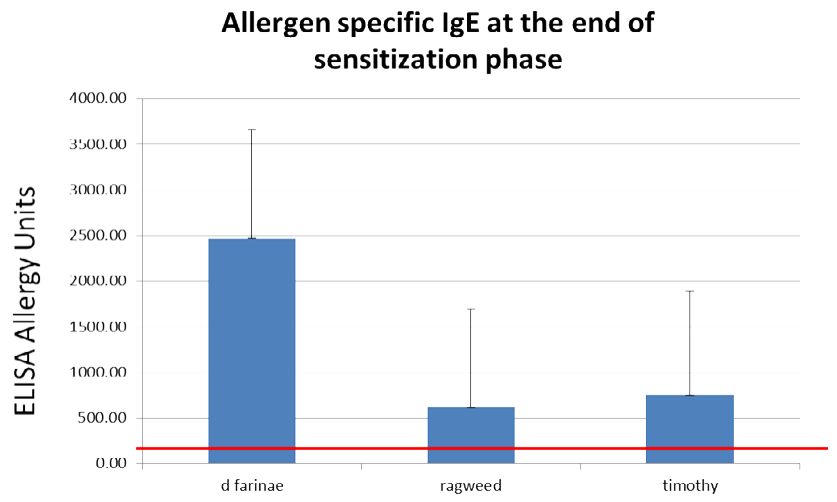

Figure 4: Mean and standard deviations of allergen specific $\lg E$ for the three allergens of reference of this study at the end of the sensitization phase. A result higher than 150 Allergy Units was considered a positive result. The red line shows the 150 mark. 
Citation: Marsella R, Ahrens K (2013) Clinical and Immunologic Effects of Allergen-Specific Sublingual Immunotherapy in a Canine Model of Atopic Dermatitis: A Double Blind, Randomized, Controlled Study. J Allergy Ther 4: 157. doi:10.4172/2155-6121.1000157

CADESI scores before starting SLIT phase

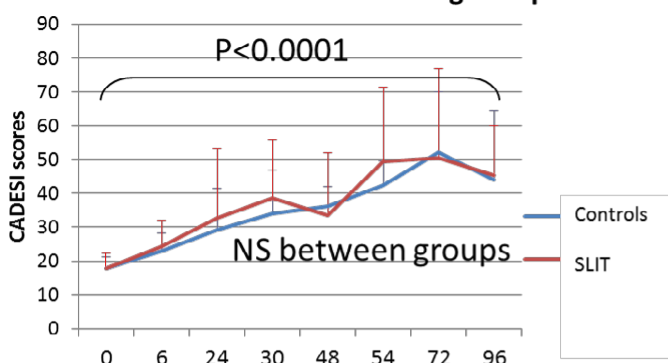

A $\quad \uparrow \quad \uparrow \quad \uparrow \quad \begin{array}{cccc}\text { Hours } \\ \text { Post Challenge }\end{array}$

Controls $(n=6)$

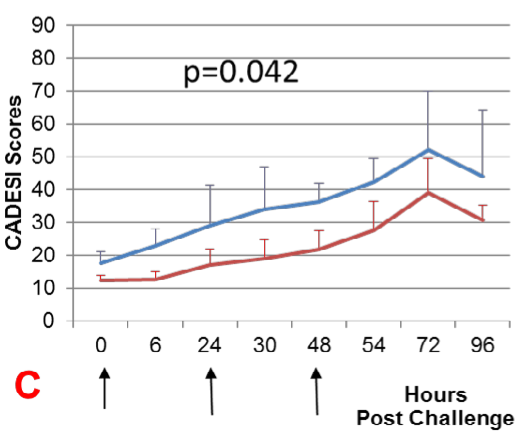

CADESI scores at the end of SLIT phase

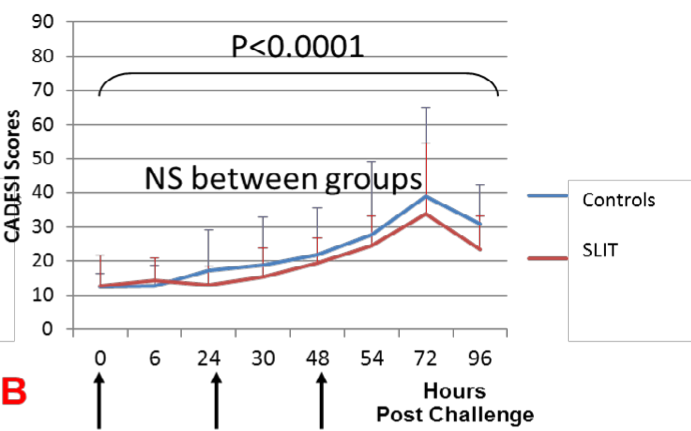

$\operatorname{SLIT}(n=12)$

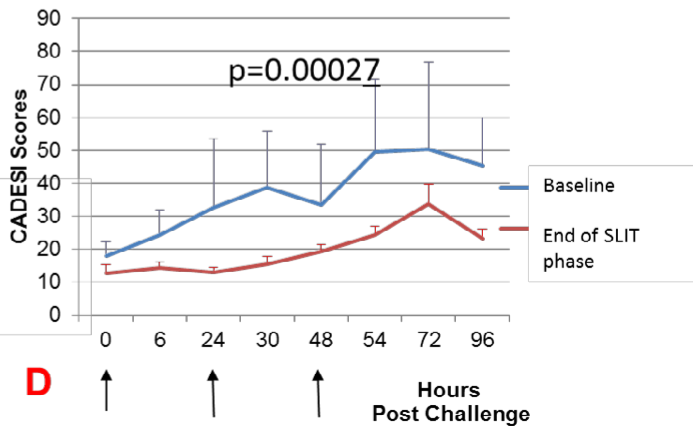

Figure 5: Total CADESI (Canine Atopic dermatitis Extent and Severity Index Score) scores and standard deviation of SLIT and control groups in the course of allergen challenge before the beginning of SLIT phase (5A) and after 12 months of SLIT (5B). The arrows indicate the time points of application of allergens. Clinical scores were not significantly different between the two groups (NS, no significant effect of group on ANOVA) at month 0 or baseline (5A) and at month 12 at the end of SLIT phase (5B). When the comparison was done within each group before and after SLIT, a statistically significant decrease was found both in the contro $(p=0.042,5 C)$ and in the SLIT group $(p=0.00027,5 D)$.
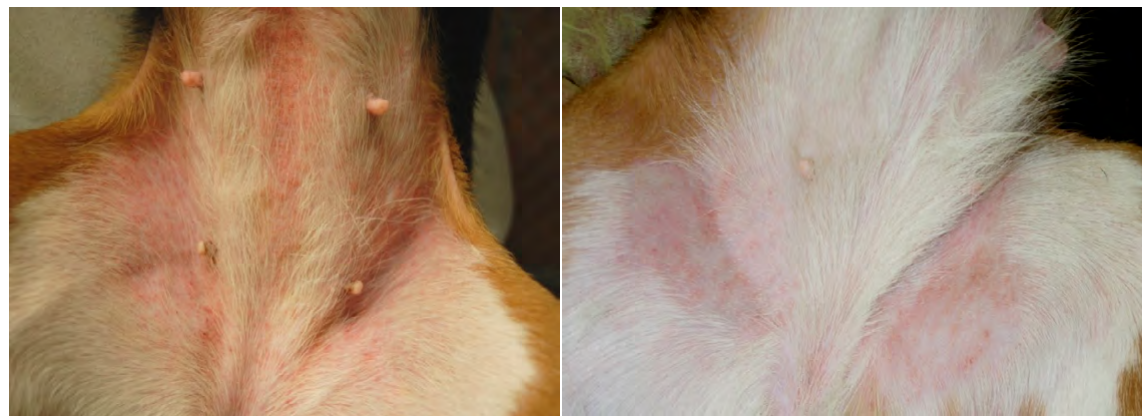

Figure 6: Clinical lesions during the allergen challenge in one of the SLIT treated Beagles at baseline (on the left) and after 12 months of SLIT (on the right). These pictures were taken 24 hours after allergen exposure and show the erythematous macular and papular eruption triggered by the allergen exposure. In the picture on the right although the papules are still visible, the erythema is markedly decreased.

evidence of oral pruritus or urticaria was found during the study. No facial or oral pruritus was noted.

Allergen specific IgE: For allergen-specific IgE to D. farinaea 2-Group x 5-Time REML ANOVA showed a significant effect of time ( $\mathrm{p}=0.0242$; end $<$ beginning), but no effect of group or group $\mathrm{x}$ time interaction (Figure 8). For allergen-specific IgE to RW ANOVA showed an effect of time ( $\mathrm{p}=0.0074$, end $>$ beginning) but no effect of group or group $\times$ time interaction (Figure 9 ). No significant differences were found for allergen specific IgE to TG (data not shown).
TGF- $\beta$ : For TGF- $\beta 1$, a significant increase was found for RW in the SLIT group after 12 months of treatment $(p=0.03)$ while no changes were observed in the control group (Figure 10). This increase was abated 2 months after the end of SLIT suggesting that it is not long lasting after discontinuation of SLIT and that the change was directly caused by SLIT. No other significant findings were detected for TGF- $\beta 1$ after stimulation with the other allergens (data not shown).

IL-10: After 12 months of SLIT a significant increase of RW induced IL-10 was found. ANOVA showed a significant effect of group $(\mathrm{p}=0.03$, 


\section{CADESI scores without allergen exposure}

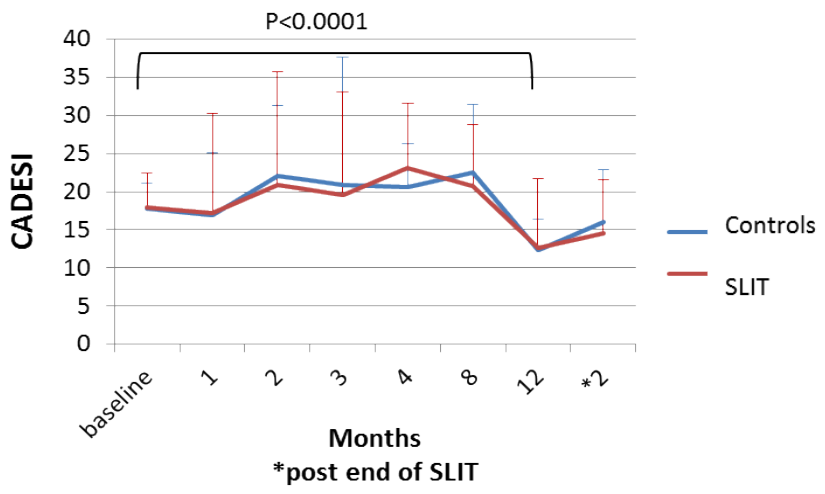

Figure 7: Total CADESI (Canine Atopic dermatitis Extent and Severity Index Score) scores and standard deviations without allergen stimulation for both SLIT and control groups. No statistically significant difference was found between groups but a significant effect of time was found comparing scores at baseline with scores at month $12(p<0.001)$. These scores reflected the severity of clinical signs without any acute challenge with allergens.

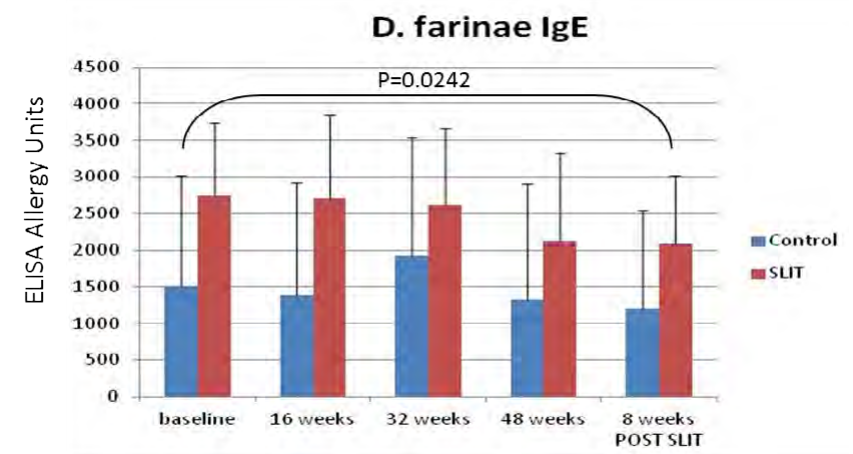

Figure 8: Allergen specific IgE for $D$. farinae the two groups over the course of the SLIT phase. Values are expressed as means and standard deviations. ANOVA showed a significant effect of time $(p=0.0242)$ but no significant effect of group.

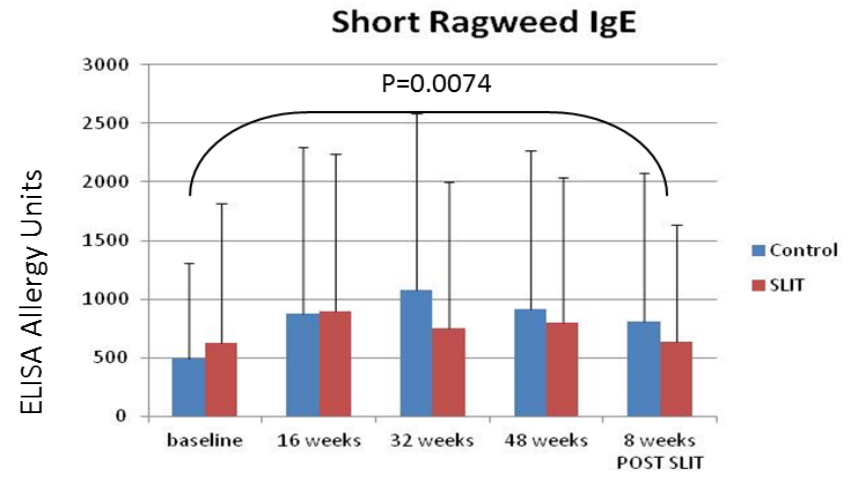

Figure 9: Allergen specific IgE for ragweed over the course of the SLIT study expressed as means and standard deviations. An effect of time $(p=0.0074$, end $>$ beginning) but no significant effect of group or group by time was found by the ANOVA.

SLIT $>$ control) and time ( $\mathrm{p}=0.0009$, end $>$ beginning) as shown in Figure 11. Interestingly, TG induced IL-10 increased for both groups at the end of the study $(\mathrm{p}<.0001)$. ANOVA also showed a significant group $\times$ time interaction with the control group spiking 2 months after stopping SLIT and reaching higher values than the SLIT group (Figure 12). No significant differences were found for IL-10 after D. farinae stimulation (data not shown).

\section{Discussion}

This study is the first vehicle controlled study to evaluate the efficacy of SLIT in a canine model for AD and due to its small size is considered a pilot study. The percentage decrease of clinical signs found in the SLIT group using our experimental model (48\%) is similar to what is reported in human medicine using SLIT. A $45 \%$ decrease was described for the active group in a vehicle controlled study using patients with rhinoconjunctivitis [30] and 46\% reduction of clinical scores was reported in an open non-controlled trial in patients with $\mathrm{AD}$ sensitive to dust mites [31] after one year of SLIT. Some of the published studies also report on the decreased need for rescue medications [32]

\section{TGF-beta RW}

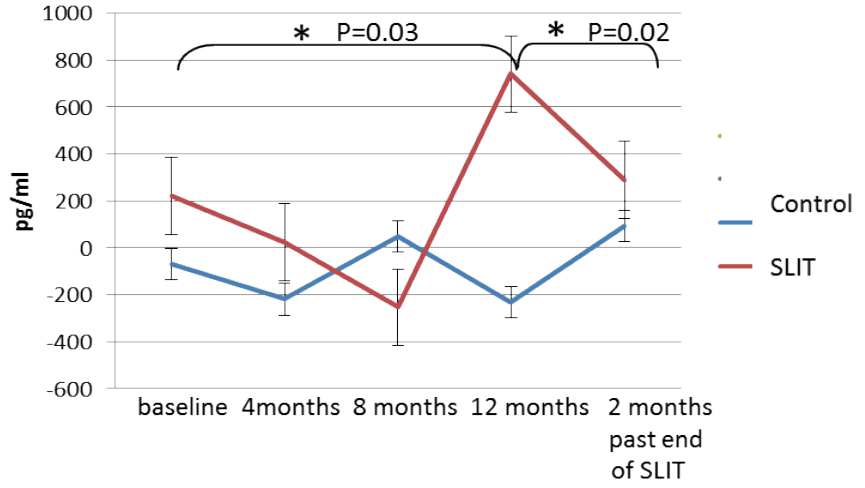

Figure 10: A significant $\left(^{*}\right)$ increase of ragweed induced TGF- $\beta 1$ was found for the SLIT group at the end of 12 months of SLIT followed by a decrease after SLIT was discontinued. The lines show the means and the bars the standard deviations of TGF- $\beta 1$ produced by peripheral blood mononuclear cells. The changes observed in the SLIT group are consistent with a SLIT induced increase of $\mathrm{T}$ regulatory response.

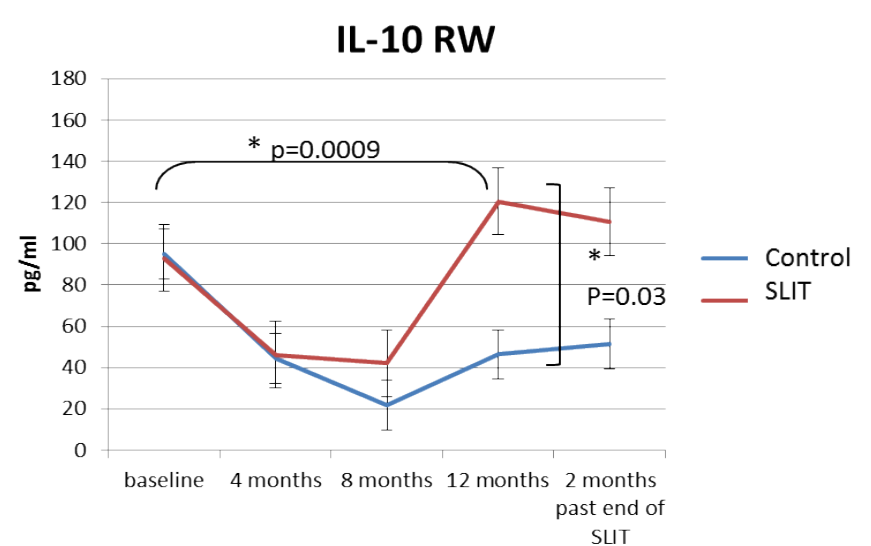

Figure 11: IL-10 produced by peripheral blood mononuclear cells after incubation with ragweed (RW) allergen expressed as means and standard deviations. A significant $\left(^{*}\right)$ increase was found for the SLIT group at the end of 12 months of SLIT. The SLIT group was significantly higher than the control group at the end of the study. 


\section{IL-10 TG}

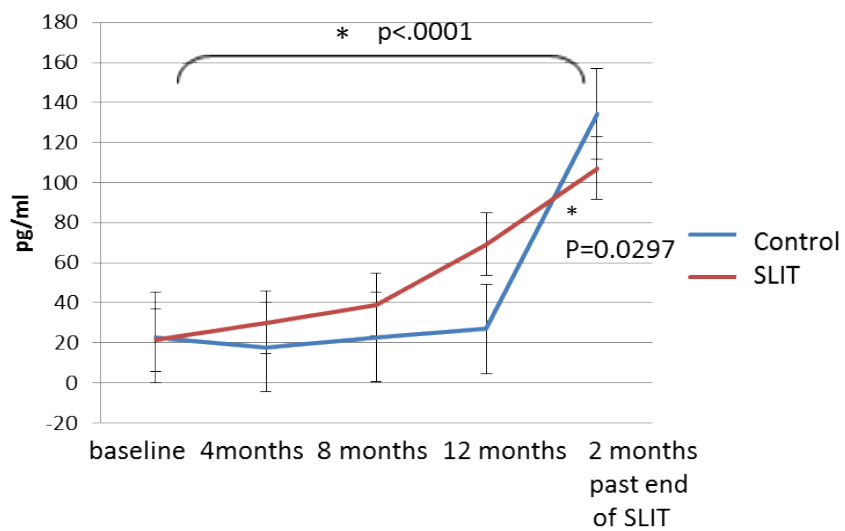

Figure 12: IL-10 produced by peripheral blood mononuclear cells after incubation with Timothy grass (TG) allergen expressed as means and standard deviations. A significant $\left({ }^{*}\right)$ increase was found at the end of the study for both the SLIT and the control group $(p<.0001)$. A significant group $x$ time interaction was also found meaning that the control and SLIT group changed in different directions 2 months after the end of the study $(p=0.0297)$.

In our study no other treatments were allowed during SLIT thus we cannot comment on the decreased need for rescue medications. Direct comparison with other studies is difficult also as some used only one allergen and the strength and protocols varied greatly across studies. This study was considered the first step toward the investigation of the suitability of this model for studies on SLIT. Future studies should be considered evaluating different protocols and dosing regimens to identify the most effective protocol as this is currently an area of controversy in human medicine.

The strength of this study relied on the controlled environment and the use of vehicle group although the number of animals used was small, due to the expenses of maintaining a colony of dogs for such an extended period of time. Also, we did not have additional groups of controls (e.g., not sensitized animals being challenged with allergens or sensitized animals being challenged with the vehicle used for the allergens). Pilot studies done during the course of the validation of this model had shown that non sensitized dogs do not develop dermatitis when challenged with the dose of allergen used for the present study [24]. For these reasons, in combination with the cost of having more controls groups, the only control that was done for the present study the one receiving the vehicle of SLIT. The decrease of clinical scores in the control group in our study is an interesting finding. This reduction could be partly due to the fact that the dogs in the vehicle group had not been exposed at all to allergens for 12 months as there was no allergen challenge scheduled during the 12 months of SLIT phase. In hindsight it would have been beneficial to schedule controlled allergen challenges during the course of SLIT to mimic a real life situation of moderate allergen exposure while administering SLIT. While the lack of allergen exposure for 12 months may have been responsible for the decrease of CADESI scores, it is important to note that it did not lead to a decrease of the allergen specific IgE, which remained in the positive range. Interestingly, other studies in humans have also reported clinical improvement in glycerin (vehicle) treated patients [33]. In one study on the effect of SLIT in children with mild to moderate asthma sensitized to DM a 30\% improvement was reported in the control group after 12 months of treatment. That study was carried out for an addition 12 months and no additional improvement was detected in the glycerin group in the subsequent year. Whether this represented a placebo effect or some ability of the glycerin to either immune-modulate or to increase adhesion in the sublingual area of other allergens that can modulate the immune system is not known. The study in children did not evaluate immunologic parameters other than IgE and IgG (for which there was no difference between pre and post therapy) and primarily focused on clinical outcomes. Nevertheless, the immunologic results [31] did not show significant differences in DM-specific IgE and total DM-specific IgG or IgG4 between the active and placebo groups. On our study we did not measure IgG4, as the role of this subtype of IgG has not been well characterized in veterinary medicine or confirmed in the more recent literature.

In terms of immunological parameters, allergen-specific immunotherapy has been shown to shift the response from a Th2 dominated to a Th1 $[34,35]$. There is also evidence of the importance of the induction of tolerance and a $\mathrm{T}$ regulatory response [36,37]. Individual with allergies tend to have a deficient $\mathrm{T}$ regulatory response and allergen specific immunotherapy increases it. The idea behind SLIT is that the allergen is captured within the oral mucosa by Langerhanslike dendritic cells expressing high-affinity IgE receptors, producing IL-10 and TGF- $\beta$ [38]. The oral mucosa has a restricted number of proinflammatory cells, such as mast cells, and that contributes to the safety of SLIT [39]. Thus for our study we decided to investigate the effects on IL-10 and TGF- $\beta$ as they are T regulatory dependent cytokines shown to be important in successful SLIT [40-42].

In the present study we found for some allergens, such as RW, SLIT produced a significant increase of both IL-10 and TGF- $\beta$ in the SLITT his result is consistent with a beneficial response to SLIT. In hindsight it would have been interesting to challenge the dogs with individual allergens rather than with the combination to evaluate whether the clinical response could be correlated to the immunological response observed in vitro after incubation of PBMC with individual allergens. It is interesting to note how IL-10 after RW incubation drops at 4 months in both groups, probably due to the fact that after the sensitization phase ended, exposure to the allergens was drastically reduced to nonexistent (for the control group). Overtime, the SLIT group progressively increased IL-10 production, possibly as a manifestation of an induction of $\mathrm{T}$ regulatory function. The increase in IL-10 mirrors reports in human medicine that reported on significant increase of IL-10 in birch allergic patients [43] and mite allergic children undergoing SLIT [44].

In our study, an increase of TG-induced IL-10 was found in the control group after discontinuation of the study and after the last allergen challenge. The significance of this finding is unknown. IL-10 can function both as a regulatory cytokine and a Th2 cytokine and the increase after end of SLIT may suggest a behavior asaTh2 cytokine rather than a T regulatory cytokine for this specific allergen. It would be interesting to monitor immunological parameters in the two groups one year after discontinuation of SLIT so see if long lasting effects are found. Future studies should also focus on characterization of populations of $\mathrm{T}$ regulatory cells and any correlation with clinical efficacy.

Conflicting differences were found in our study in terms of allergen specific IgE at the end of SLIT but for the most part there were no significant differences between groups. Although one proposed mechanism for SLIT is a decrease of allergen specific IgE other reports have been also published in human medicine where no significant differences in allergen specific IgE between active and control group were detected [28]. Other authors have reported on a protective effect from progressive sensitization and a less decrease of $\mathrm{IgE}$ overtime in patients undergoing SLIT [45]. It is important to add that, at least in 
Citation: Marsella R, Ahrens K (2013) Clinical and Immunologic Effects of Allergen-Specific Sublingual Immunotherapy in a Canine Model of Atopic Dermatitis: A Double Blind, Randomized, Controlled Study. J Allergy Ther 4: 157. doi:10.4172/2155-6121.1000157

human medicine, the decrease of allergen specific IgE after allergenspecific immunotherapy seems to be most detectable after years of therapy thus a lack of dramatic change after 12 months could be consistent with observations in studies in human medicine [43]. We realize that the monitoring of $\operatorname{IgE}$ is not currently considered the main mechanism of ASIT and that future studies should focus on the changes in T regulatory cells.

No adverse effects were found in our study so it is fair to say that SLIT was safe in this canine model of AD as it is typically in humans [13]. In summary, based on this pilot study using an experimental model of AD of highly reacting atopic beagles we conclude that SLIT has similar efficacy for $\mathrm{AD}$ in dogs as it has been currently reported in human medicine making this model a useful tool to further pursue mechanistic studies that can shed light on the best use of SLIT for AD.

\section{Acknowledgment}

The author would like to thank Dr. William Perlstein for the analysis of the data This study was partly self funded and partly sponsored by Nelco and Fariseau.

\section{References}

1. Eng PA, Borer-Reinhold M, Heijnen IA, Gnehm HP (2006) Twelve-year follow-up after discontinuation of preseasonal grass pollen immunotherapy in childhood. Allergy 61: 198-201.

2. Walker SM, Durham SR, Till SJ, Roberts G, Corrigan CJ, et al. (2011) Immunotherapy for allergic rhinitis. Clin Exp Allergy 41: 1177-1200.

3. Olivry T, DeBoer DJ, Favrot C, Jackson HA, Mueller RS, et al. (2010) Treatment of canine atopic dermatitis: 2010 clinical practice guidelines from the International Task Force on Canine Atopic Dermatitis. Vet Dermatol 21: 233248.

4. Durham SR, Yang WH, Pedersen MR, Johansen N, Rak S (2006) Sublingua immunotherapy with once-daily grass allergen tablets: a randomized controlled trial in seasonal allergic rhinoconjunctivitis. J Allergy Clin Immunol 117: 802809.

5. Wilson DR, Lima MT, Durham SR (2005) Sublingual immunotherapy for allergic rhinitis: systematic review and meta-analysis. Allergy 60: 4-12.

6. Penagos M, Compalati E, Tarantini F, Baena-Cagnani R, Huerta J, et al. (2006) Efficacy of sublingual immunotherapy in the treatment of allergic rhinitis in pediatric patients 3 to 18 years of age: a meta-analysis of randomized, placebocontrolled, double-blind trials. Ann Allergy Asthma Immunol 97: 141-148.

7. Bahceciler NN, Galip N (2012) Comparing subcutaneous and sublingual Ä \pm mmunotherapy: what do we know? Curr Opin Allergy Clin Immunol 12: 640647.

8. Pajno GB, Finegold I (2011) SIT beyond respiratory diseases. Ann Allergy Asthma Immunol 107: 395-400.

9. Vanbervliet B, Tourdot S, Mascarell L, Rouzaire P, Vocanson M, et al. (2012) SLIT prevents the development of eczema in percutaneous allergen-sensitized mice. J Invest Dermatol 132: 244-246.

10. Cox L, Compalati E, Kundig T, Larche M (2013) New directions in immunotherapy. Curr Allergy Asthma Rep 13: 178-195

11. Compalati E, Rogkakou A, Passalacqua G, Canonica GW (2012) Evidences of efficacy of allergen immunotherapy in atopic dermatitis: an updated review. Curr Opin Allergy Clin Immunol 12: 427-433.

12. Eifan AO, Akkoc T, YildizA, Keles S, Ozdemir C, et al. (2010) Clinical efficacy and immunological mechanisms of sublingual and subcutaneous immunotherapy in asthmatic/rhinitis children sensitized to house dust mite: an open randomized controlled trial. Clin Exp Allergy 40: 922-932.

13. Burks AW, Calderon MA, Casale T, Cox L, Demoly P, et al. (2013) Update on allergy immunotherapy: American Academy of Allergy, Asthma \& Immunology/ European Academy of Allergy and Clinical Immunology/PRACTALL consensus report. J Allergy Clin Immunol 131: 1288-1296.

14. Pajno GB, Caminiti L, Vita D, Barberio G, Salzano G, et al. (2007) Sublingual immunotherapy in mite-sensitized children with atopic dermatitis: a randomized, double-blind, placebo-controlled study. J Allergy Clin Immunol 120: 164-170.
15. Sikora JM, Tankersley MS; ACAAI Immunotherapy and Diagnostics Committee (2013) Perception and practice of sublingual immunotherapy among practicing allergists in the United States: a follow-up survey. Ann Allergy Asthma Immuno 110: 194-197.

16. Moran TP, Vickery BP, Burks AW (2013) Oral and sublingual immunotherapy for food allergy: current progress and future directions. Curr Opin Immunol 25 781-787.

17. Passalacqua G, Compalati E, Canonica GW (2010) Sublingual Immunotherapy: Clinical Indications in the WAO-SLIT Position Paper. World Allergy Organ J 3 : 216-219.

18. Bae JM, Choi YY, Park CO, Chung KY, Lee KH (2013) Efficacy of allergenspecific immunotherapy for atopic dermatitis: a systematic review and metaanalysis of randomized controlled trials. J Allergy Clin Immunol 132: 110-117.

19. Marsella R, Girolomoni G (2009) Canine models of atopic dermatitis: a usefu tool with untapped potential. J Invest Dermatol 129: 2351-2357.

20. Marsella R, Olivry T, Carlotti DN; International Task Force on Canine Atopic Dermatitis (2011) Current evidence of skin barrier dysfunction in human and canine atopic dermatitis. Vet Dermatol 22: 239-248.

21. Marsella R, Olivry T, Nicklin C, Lopez J (2006) Pilot investigation of a model for canine atopic dermatitis: environmental house dust mite challenge of high-lgEproducing beagles, mite hypersensitive dogs with atopic dermatitis and normal dogs. Vet Dermatol 17: 24-35.

22. Marsella R, Samuelson D, Doerr K (2010) Transmission electron microscopy studies in an experimental model of canine atopic dermatitis. Vet Dermatol 21 $81-88$

23. Marsella R, Olivry T, Maeda S (2006) Cellular and cytokine kinetics after epicutaneous allergen challenge (atopy patch testing) with house dust mites in high-lgE beagles. Vet Dermatol 17: 111-120

24. Olivry T, Marsella R, Iwasaki T, Mueller R; International Task Force On Canine Atopic Dermatitis (2007) Validation of CADESI-03, a severity scale for clinical trials enrolling dogs with atopic dermatitis. Vet Dermatol 18: 78-86.

25. Arbes SJ Jr, Gergen PJ, Elliott L, Zeldin DC (2005) Prevalences of positive skin test responses to 10 common allergens in the US population: results from the third National Health and Nutrition Examination Survey. J Allergy Clin Immunol 116: 377-383.

26. Warm K, Lindberg A, Lundbäck B, Rönmark E (2013) Increase in sensitization to common airborne allergens among adults -- two population-based studies 15 years apart. Allergy Asthma Clin Immunol 9: 20.

27. Marsella R (2009) Evaluation of Lactobacillus rhamnosus strain GG for the prevention of atopic dermatitis in dogs. Am J Vet Res 70: 735-740.

28. Marsella R, Saridomichelakis MN (2010) Environmental and oral challenge with storage mites in beagles experimentally sensitized to Dermatophagoides farinae. Vet Dermatol 21: 105-111.

29. StrasserA, KalmarE, NiedermüllerH(1998)Asimple method for the simultaneous separation of peripheral blood mononuclear and polymorphonuclear cells in the dog. Vet Immunol Immunopathol 62: 29-35.

30. PurelloD'Ambrosio F, Sangemi S, Isola S, La Motta N, Ricciardi L, et al. (1999) Sublingual immunotherapy: a double-blind, placebo-controlled trial with Parietariajudaica extract standardised in mass units in patients with rhinoconjunctivitis, asthma or both. Allergy 54: 968-973.

31. Cadario G, Galluccio AG, Pezza M, Appino A, Milani M, et al. (2007) Sublingual immunotherapy efficacy in patients with atopic dermatitis and house dust mites sensitivity: a prospective pilot study. Curr Med Res Opin 23: 2503-2506.

32. Qin YE, Mao JR, Sang YC, Li WX (2013) Clinical efficacy and compliance of sublingual immunotherapy with Dermatophagoides farinae drops in patients with atopic dermatitis. Int J Dermatol .

33. Pajno GB, Morabito L, Barberio G, Parmiani S (2000) Clinical and immunologic effects of long-term sublingual immunotherapy in asthmatic children sensitized to mites: a double-blind, placebo-controlled study. Allergy 55: 842-849.

34. Yacoub MR, Colombo G, Marcucci F, Caminati M, Sensi L, et al. (2012) Effects of sublingual immunotherapy on allergic inflammation: an update. Inflamm Allergy Drug Targets 11: 285-291

35. Ciprandi G, Tosca MA, Marseglia GL (2009) Sublingual immunotherapy mechanisms of action: the role of Th1 response. Int J Immunopathol Pharmaco 22: $9-12$. 
Citation: Marsella R, Ahrens K (2013) Clinical and Immunologic Effects of Allergen-Specific Sublingual Immunotherapy in a Canine Model of Atopic Dermatitis: A Double Blind, Randomized, Controlled Study. J Allergy Ther 4: 157. doi:10.4172/2155-6121.1000157

Page 9 of 9

36. Maggi E, Vultaggio A, Matucci A (2012) T-cell responses during allergenspecific immunotherapy. Curr Opin Allergy Clin Immunol 12: 1-6.

37. Scadding GW, Shamji MH, Jacobson MR, Lee DI, Wilson D, et al. (2010) Sublingual grass pollen immunotherapy is associated with increases in sublingual Foxp3-expressing cells and elevated allergen-specific immunoglobulin G4, immunoglobulin A and serum inhibitory activity for immunoglobulin E-facilitated allergen binding to B cells. Clin Exp Allergy 40: 598-606.

38. Moingeon P, Batard T, Fadel R, Frati F, Sieber J, et al. (2006) Immune mechanisms of allergen-specific sublingual immunotherapy. Allergy 61: 151165.

39. O'Hehir RE, Sandrini A, Anderson GP, Rolland JM (2007) Sublingual allergen immunotherapy: immunological mechanisms and prospects for refined vaccine preparation. Curr Med Chem 14: 2235-2244.

40. Nouri-Aria KT, Wachholz PA, Francis JN, Jacobson MR, Walker SM, et al. (2004) Grass pollen immunotherapy induces mucosal and peripheral IL-10 responses and blocking IgG activity. J Immunol 172: 3252-3259.
41. Akdis M, Blaser K, Akdis CA (2005) T regulatory cells in allergy: novel concepts in the pathogenesis, prevention, and treatment of allergic diseases. J Allergy Clin Immunol 116: 961-968.

42. Woodfolk JA (2005) High-dose allergen exposure leads to tolerance. Clin Rev Allergy Immunol 28: 43-58.

43. Guida G, Boita M, Scirelli T, Bommarito L, Heffler E, et al. (2012) Innate and lymphocytic response of birch-allergic patients before and after sublingual immunotherapy. Allergy Asthma Proc 33: 411-415.

44. Barberi S, Villa MP, Pajno GB, La Penna F, Barreto M, et al. (2011) Immune response to sublingual immunotherapy in children allergic to mites. J Biol Regul Homeost Agents 25: 627-634.

45. Baron-Bodo V, Batard T, Nguyen H, Fréreux M, Horiot S, et al. (2012) Absence of IgE neosensitization in house dust mite allergic patients following sublingual immunotherapy. Clin Exp Allergy 42: 1510-1518. 